\title{
Interrelated Atrioventricular Reentrant Tachycardia and Idiopathic Left Ventricular Tachycardia in a Patient with Manifested Bystander Accessory Pathway
}

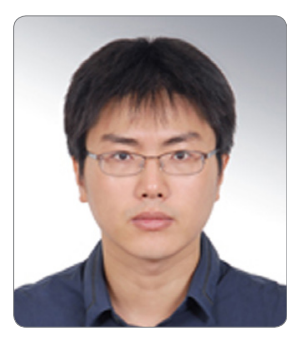

Da Hyon Lee
Da Hyon Lee, MD; Seung Jung Park, MD, PhD; Young Keun On, MD, PhD; June Soo Kim, MD, PhD; Kyoung Min Park, MD, PhD

Division of Cardiology, Department of Internal Medicine, Heart Vascular and Stroke Institute, Samsung Medical Center, Sungkyunkwan University School of Medicine, Seoul, Republic of Korea.

Received: February 16, 2017

Revision Received: March 20, 2017

Accepted: May 21, 2017

Correspondence: Kyoung Min Park, MD, PhD

Division of Cardiology, Department of Internal Medicine, Heart Vascular and Stroke Institute,

Samsung Medical Center, Sungkyunkwan

University School of Medicine, 81 Irwon-ro,

Gangnam-gu, Seoul 06351, Republic of Korea

Tel: +82-2-3410-3419 Fax: +82-2-3410-3419

E-mail: kyoungmin.park@samsung.com

Copyright (C) 2017 The Official Journal of Korean Heart Rhythm Society Editorial Board \& MMK Co., Ltd.

\begin{abstract}
Double tachycardia is defined as the coexistence of supraventricular tachycardia (SVT) and ventricular tachycardia (VT). In clinical practice, incidentally encountered double tachycardia is difficult to diagnose during electrophysiology study without prior documentation of both tachycardias. SVT can be confused with VT because SVT sometimes show an aberrant conduction depending on heart rate. In the present case, a patient with Wolff-Parkinson-White syndrome exhibited atrioventricular reentrant tachycardia (AVRT) via a concealed bypass tract and idiopathic left ventricular tachycardia (ILVT), which were difficult to discriminate because of their similar cycle length and interrelationship.
\end{abstract}

Key Words: - Atrioventricular Reentrant Tachycardia - Idiopathic Left Ventricular Tachycardia - Double Tachycardia

\section{Introduction}

Double tachycardia is defined as the coexistence of supraventricular tachycardia (SVT) and ventricular tachycardia (VT), and was initially reported in patients with left ventricular dysfunction or digoxin intoxication. ${ }^{1-4}$ Since then, exercise- or catecholamine-induced double tachycardia has been reported.5,6 Recently, double tachycardia in patients with no obvious structural heart disease has been repeatedly noted. The most common type of double tachycardia is atrioventricular nodal reentrant tachycardia (AVNRT) and idiopathic outflow tract VT. $^{7-9}$ On the other hand, double tachycardia consisting of atrioventricular reentrant tachycardia (AVRT) and idiopathic left ventricular tachycardia (ILVT) is relatively rare and has been documented in only two case reports and in one observational study. In the present case, a patient with Wolff-Parkinson-White (WPW) syndrome exhibited AVRT and ILVT, which transformed from one morphology to another on electrophysiology testing and was cured with consecutive ablations of two accessory pathways and a mid-inferior septum.

\section{Case}

A 40-year-old man with WPW syndrome had recurrent episodes of palpitation over the course of 10 years. He had taken only prophylactic oral verapamil $(180 \mathrm{mg} /$ day $)$ and bisoprolol 
$(1.5 \mathrm{mg} /$ day) for paroxysmal supraventricular tachycardia (PSVT) without having undergone an electrophysiology study at his original hospital. On surface electrocardiography (ECG) during sinus rhythm, ventricular pre-excitation with a negative delta wave was observed in leads $\mathrm{V}_{1}$, III, and aVF; and a positive delta wave, in other leads, which suggested the presence of a right posteroseptal accessory pathway (AP; Figure 1). Finally, he was referred to the Samsung Medical Center because of recurrent palpitations despite being on medication. No structural heart abnormalities were detected on standard admission examination, which included echocardiography.

An electrophysiology study was performed in an antiarrhythmic drug-free state. Two quadripolar catheters were placed in the His bundle region and at the right ventricular apex under local anesthesia. The high right atrium (HRA) and coronary sinus (CS) were mapped with a duo-decapolar catheter. The earliest ventricular activation site during normal sinus rhythm was observed at the proximal recording site of the CS catheter, which suggested right posteroseptal AP, as expected on surface ECG (Figure 2A). The earliest ventricular activation site during ventricular pacing was observed at the distal recording site of the CS catheter, which suggested left lateral AP (Figure 2A). AVRT via the left lateral AP (cycle length: $404 \mathrm{msec}$ ) was spontaneously induced in the middle of catheter manipulation. Subsequently, the
AVRT rhythm spontaneously converted to wide QRS tachycardia of a similar cycle length ( $410 \mathrm{msec})$, with retrograde conduction via the left lateral AP (Figure 2B). After rapid ventricular pacing was performed to terminate the AVRT, the AVRT rhythm transformed into atrial fibrillation (AF) with irregularly narrow QRS complexes, which gradually changed to regularly wide QRS tachycardia with multiple irregular HRA signals. Intracardiac cardioversion $(10 \mathrm{~J})$ was conducted to terminate AF. AF was terminated after cardioversion, but the regularly wide QRS with eccentric retrograde conduction continued. At that time, we considered this wide QRS tachycardia as AVRT with aberrant conduction; thus, we applied radiofrequency (RF) energy at the earliest atrial activation site via a trans-septal approach to terminate the AVRT by using the left lateral AP. Consequently, retrograde ventriculoatrial (VA) conduction via the left lateral AP was blocked, but wide QRS tachycardia was sustained. Typical atrioventricular (AV) dissociation was also noted (Figure 2C). Only then did we realize that the sustained wide QRS rhythm was VT with retrograde conduction via the left lateral AP. More specifically, we considered it ILVT on the basis of the right bundle branch block (RBBB) pattern with left axis deviation on ECG (Figure 2D). Four applications of RF energy were made at the mid-inferior septum via a retrograde aortic approach targeting the earliest Purkinje potential (Figure 3A, B), and then VT was

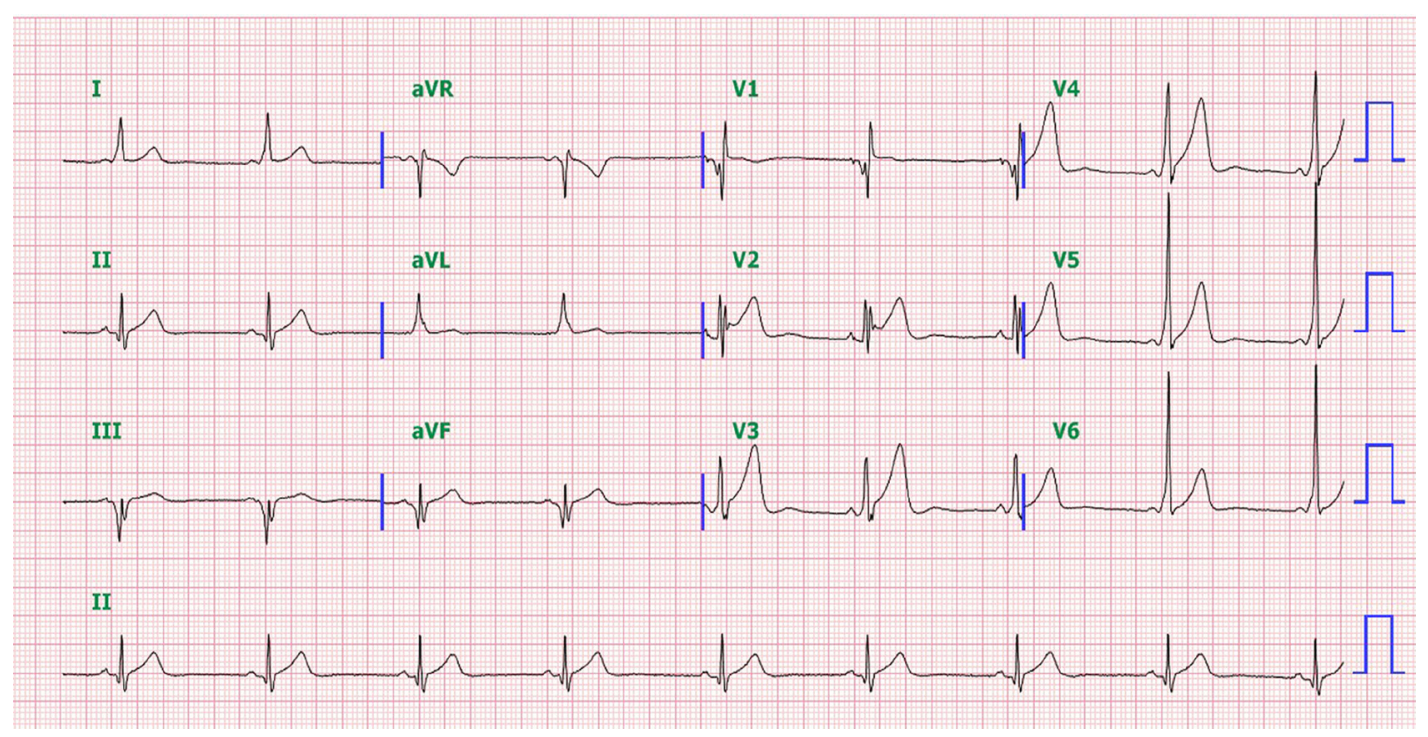

Figure 1. A 12-lead ECG during sinus rhythm showed ventricular pre-excitation with a negative delta wave in leads $V_{1}$, III, and aVF and a positive delta wave in the other leads. It suggests the presence of a right posteroseptal accessory pathway. 


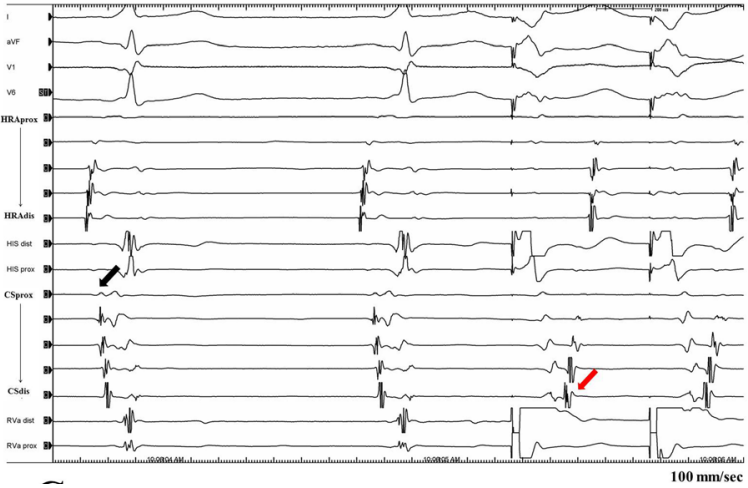

C

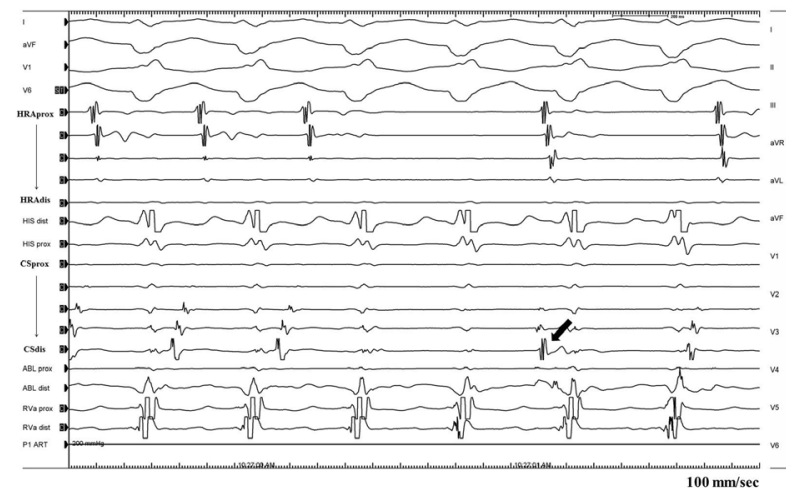

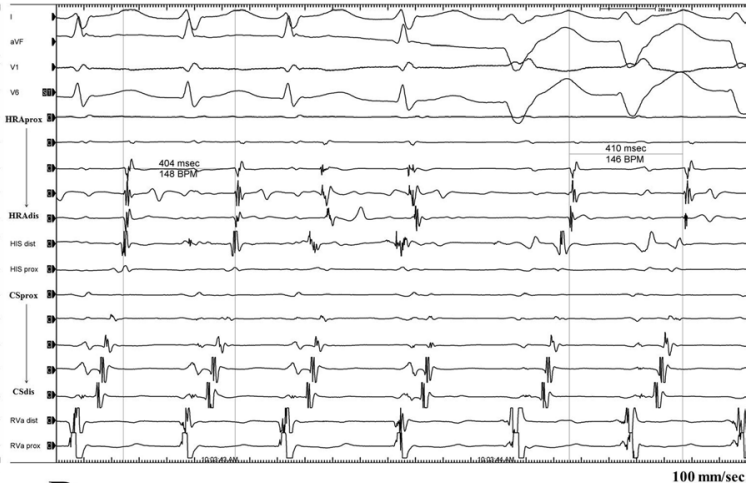

D

Figure 2. Intracardiac electrogram (EGM) and surface ECG images of transformed double tachycardia. (A) Baseline EGM during sinus rhythm and ventricular pacing. The earliest ventricular activation site during sinus rhythm was observed at the proximal recording site of the coronary sinus (CS) catheter, which suggested right posteroseptal accessory pathway (AP) (black arrow). The earliest ventricular activation site during ventricular pacing was observed at the distal recording site of the CS catheter, which suggested left lateral AP (red arrow). (B) An initial narrow QRS tachycardia (cyclic length: $404 \mathrm{msec}$ ) with retrograde atrial conduction via the left lateral AP spontaneously converted to wide QRS tachycardia (cyclic length: $410 \mathrm{msec}$ ) with retrograde atrial conduction via the left lateral AP. (C) The eccentric retrograde atrial conduction disappeared after ablation of the left lateral AP, but wide QRS tachycardia continued with typical atrioventricular dissociation (arrow). (D) ECG image of the wide QRS tachycardia showing a right bundle branch block pattern with left axis deviation on ECG. It means that wide QRS tachycardia is ventricular tachycardia with eccentric atrial activation, especially idiopathic left ventricular tachycardia based on ECG morphology.

HRA, high right atrium; HIS, His bundle; CS, coronary sinus; RVa, right ventricle apex; ABL, ablation

terminated. Following ablation of this lesion, neither VT nor AVRT was provoked by extra-stimuli or rapid pacing with or without isoproterenol infusion. In addition, we ablated the manifested posteroseptal bystander AP, and the delta wave on ECG disappeared.

\section{Discussion}

Double tachycardia is a relatively uncommon arrhythmia. However, recently, several case reports and observational studies have reported double tachycardia with AVNRT and outflow tract tachycardia. ${ }^{7-9}$ Compared with the combination of AVNRT and outflow tract VT, the frequency of combined AVRT and ILVT is quite scarce. Watanabe et al. first reported a case of coexisting AVRT from a concealed left lateral AP and ILVT from a left anterior fascicle, which transformed from one tachycardia to another. ${ }^{10}$ Moreover, Lim et al. introduced a case of WPW syndrome that showed separately induced AVRT from a left lateral AP and ILVT from a left posterior fascicle on an electrophysiology study. ${ }^{11}$ Recently, $\mathrm{Hu}$ et al. published seven patients who simultaneously showed AVRT via a concealed pathway among 140 patients with documented ILVT originating 
A

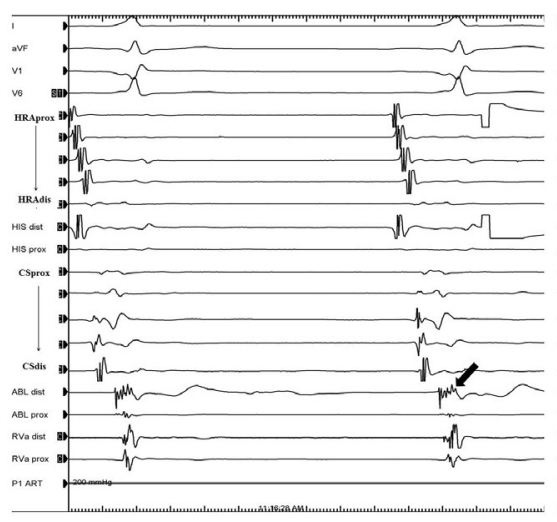

B

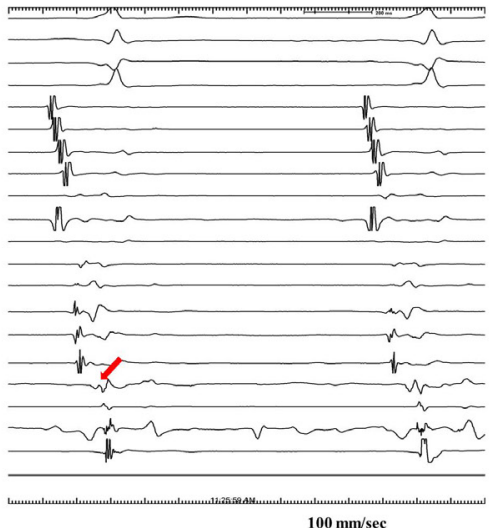

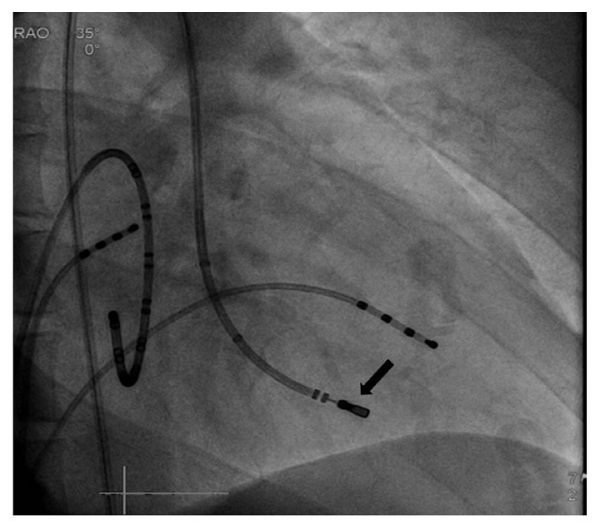

Figure 3. Intracardiac electrogram of the idiopathic left ventricular tachycardia (ILVT) ablation and fluoroscopic $30^{\circ}$ right anterior oblique projection of the ablation catheter. (A) The ablation of the ILVT was guided by the presence of Purkinje potential (black arrow), which disappeared (red arrow) after four applications of radiofrequency energy. (B) In the right oblique view, the ablation catheter (arrow) is located at the mid-inferior septum of the left ventricle.

HRA, high right atrium; HIS, His bundle; CS, coronary sinus; RVa, right ventricle apex; $A B L$, ablation

from the left posterior fascicle. ${ }^{12}$ In our case, interrelated AVRT and ILVT were demonstrated during an electrophysiology study.

Double tachycardia can be difficult to recognize if both types of tachycardia are not documented previously on ECG or if the signal patterns on electrophysiology study are similar, such as AVRT and VT with eccentric atrial activation. When wide QRS tachycardia with eccentric atrial activation is observed on electrophysiology study, orthodromic AVRT with bundle branch block or aberrant conduction, antidromic AVRT, and VT with retrograde atrial conduction have to be considered as possible differential diagnoses. Methods such as rapid atrial pacing for atrioventricular ventriculoatrial (AVVA) response and AV dissociation, ${ }^{12}$ or the comparison of cyclic length ${ }^{11}$ or AP ablation during tachycardia can be useful for the differential diagnosis of wide QRS tachycardia with eccentric atrial activation. In this case, VT with an RBBB pattern showed a similar cycle length to that of the previous AVRT. Consequently, AVRT with an aberrant $\mathrm{RBBB}$ conduction pattern using an ipsilateral AP was first suspected in accordance with Coumel's law. ${ }^{13}$ Thus, we could not recognize the sustained monomorphic VT with retrograde atrial activation through the bypass tract until successfully ablating the left lateral AP. Furthermore, if we had ablated the left lateral AP targeting the eccentric VA conduction during ventricular pacing in sinus rhythm, we could have missed the ILVT because it was reported that VT may not be provoked after AP ablation in double tachycardia composed of AVRT and ILVT.12

In our case, double tachycardia was especially difficult to identify because AVRT and ILVT were interrelated during the electrophysiology study. Atrial fibrillation and AVRT transformed to ILVT spontaneously. Although uncommon, atrial pacing was already shown to induce VT, ${ }^{14}$ and SVT was reported to be the trigger of VT with a reentry mechanism. ${ }^{15}$ This suggests that VT can be prevented by SVT ablation, especially in patients with tachycardia-induced tachycardia. However, the ablation strategy in patients with double tachycardia has not been standardized, and recent studies reported that either ablation of double tachycardia did not prevent another tachycardia induction, ${ }^{8,16}$ even in cases of tachycardia-induced tachycardia. ${ }^{10,17}$ In our case, unplanned ablation of both arrhythmic substrates was performed because of persistent VT after the ablation of the left lateral AP. Large-scale trials are needed to establish the standard ablation strategy in interrelated double tachycardia.

To our knowledge, this is the first case that describes interrelated AVRT and ILVT in a patient with manifested bystander AP. In addition, the coexistence of AVRT and ILVT can be diagnostically challenging, especially in patients without a documented wide QRS tachycardia on ECG or with tachycardiainduced tachycardia. Therefore, a detailed electrophysiology study 
should be required when taking into consideration the possibility of double tachycardia consisting of AVRT and ILVT, even in patients with WPW syndrome and documented PSVT on ECG.

\section{Acknowledgements}

None.

\section{References}

1) Jordan RM, McAnulty JH, Ritzmann L. Alternating atrial and ventricular tachycardia. Br Heart J. 1979;41:734-737.

2) Wishner SH, Kastor JA, Yurchak PM. Double atrial and atrioventricular junctional tachycardia. $N$ Engl $\mathrm{J}$ Med. 1972;287:552-553.

3) Halkin H, Kaplinsky E. Simultaneous tachycardias associated with acute myocardial infarction. Chest. 1971;60:394-396.

4) Chowdhry IH, Hariman RJ, Gomes JA, El-Sherif N. Transient digitoxic double tachycardia. Chest. 1983;83:686-687.

5) Eldar M, Belhassen B, Hod H, Schuger CD, Scheinman MM. Exercise-induced double (atrial and ventricular) tachycardia: a report of three cases. J Am Coll Cardiol. 1989;14:1376-1381.

6) Benson DW, Gallagher JJ, Sterba R, Klein GJ, Armstrong BE. Catecholamine induced double tachycardia: case report in a child. Pacing Clin Electrophysiol. 1980;3:96-103.

7) Zardini M, Boyle NG, Josephson ME. Coexistent narrow and wide QRS complex tachycardia: an interesting duo. Pacing Clin Electrophysiol. 1996;19:363-366.

8) Kautzner J, Cihák R, Vancura V, Bytesník J. Coincidence of idiopathic ventricular outflow tract tachycardia and atrioventricular nodal reentrant tachycardia. Europace. 2003;5:215-220.

9) Cooklin M, McComb JM. Tachycardia induced tachycardia: case report of right ventricular outflow tract tachycardia and AV nodal reentrant tachycardia. Heart. 1999;81:321-322.

10) Watanabe I, Kunimoto S, Kondo K, Kojima T, Nakai T, Shindo A, Oshikawa N, Saito S, Ozawa Y, Kanmatsuse K. Radiofrequency catheter ablation of coexistent atrioventricular reciprocating tachycardia and left ventricular tachycardia originating in the left anterior fascicle. Jpn Circ J. 1999;63:223-227.

11) Lim HE, Pak H-N, Kim Y-H. Coexisting idiopathic left ventricular tachycardia and atrioventricular reentrant tachycardia in a patient with Wolff-Parkinson-White syndrome. Pacing Clin Electrophysiol. 2009;32:265-268.

12) Hu J, Ma J, Yang Q, Liao Z, Hou Y, Zhang S. The characteristics of verapamil-sensitive idiopathic left ventricular tachycardia combined with a left accessory pathway and the effect of radiofrequency catheter ablation. Europace. 2012;14:703-708.

13) Coumel P, Attuel P. Reciprocating tachycardia in overt and latent preexcitation. Influence of functional bundle branch block on the rate of the tachycardia. Eur J Cardiol. 1974;1:423-436.

14) Brembilla-Perrot B, Terrier de La Chaise A, Shandel C. Characteristics and prognosis of ventricular tachycardia induced by atrial fibrillation. Arch Mal Coeur Vaiss. 1992;85:1291-1297.

15) Wagshal AB, Mittleman RS, Schuger CD, Huang SK. Coincident idiopathic left ventricular tachycardia and atrioventricular nodal reentrant tachycardia: control by radiofrequency catheter ablation of the slow atrioventricular nodal pathway. Pacing Clin Electrophysiol. 1994;17:386-396.

16) Kazemi B, Arya A, Haghjoo M, Sadr-Ameli MA. Coincident atrioventricular nodal reentrant and idiopathic ventricular tachycardia. Asian Cardiovasc Thorac Ann. 2006;14:284-288.

17) Weng K-P, Chiou C-W, Kung M-H, Lin C-C, Hsieh K-S. Radiofrequency catheter ablation of coexistent idiopathic left ventricular tachycardia and atrioventricular nodal reentrant tachycardia. J Chin Med Assoc. 2005;68:479-483. 